\title{
Optically Reconfigurable 1x4 Remote Node Switch for Access Networks
}

\author{
Martijn Tassaert, Student Member, IEEE, Gunther Roelkens, Member, IEEE, Harm J.S. Dorren, \\ Member, IEEE, and Oded Raz, Member, IEEE
}

\begin{abstract}
In this paper we demonstrate an optically controlled $1 \times 4$ remote node switch, based on membrane InP switches bonded to a silicon-on-insulator circuit. We show that the switch exhibits cross talk better than $25 \mathrm{~dB}$ between the output ports, and that the switch operates without receiver sensitivity penalty. Furthermore, the proposed switch architecture allows for optical clock distribution as a means to avoid the need for clock recovery at the receiver side. This is demonstrated in a proof-of-principle experiment where data and clock are sent through a single membrane InP switch.
\end{abstract}

Index Terms-Integrated optics devices; Packet switching; Alloptical networks

\section{INTRODUCTION}

$\mathrm{T}$ HE exponential increase in demand for bandwidth [1] is forcing access networks to scale up the transmission speeds they can support. These systems are traditionally based on time division multiplexing (TDM) and passive optical splitters, and are therefore struggling to keep up with the rise in line-rate, as this cuts away link budget and therefore splitting ratio and network reach [2]. One possible solution to this problem is the use of wavelength division multiplexing combined with passive splitters (WDM-PON) [3] to increase the number of subscribers and their share of the total network bandwidth, while using low loss optical demultiplexers. This way some flexibility can be obtained in the allocation of bandwidth, but users linked to a certain wavelength output of the demultiplexer still have to compete with other users on the same branch for bandwidth through TDM, and physically rewiring the network to double the number of wavelengths routed to a certain sub-network is not possible.

An alternative solution is to replace passive splitters with active optical switches [4, 5]. In these demonstrations, electronically controlled switches are used to choose between

Manuscript received XXXXXXXXX; revised XXXXXXXXX; accepted XXXXXXXX. Date of Publication XXXXXXX; date of current version XXXXXXXX. Martijn Tassaert acknowledges the BOF for a doctoral grant.

M. Tassaert is with Ghent University-imec, Ghent, B-9000 Belgium (e-mail: martijn.tassaert@intec.ugent.be).

G. Roelkens is with Ghent University-imec, Ghent, B-9000 Belgium (e-mail: gunther.roelkens@intec.ugent.be).

H. J. S. Dorren is with Eindhoven University of Technology, Eindhoven, The Netherlands (e-mail: hdorren@tue.nl).

O. Raz is with Eindhoven University of Technology, Eindhoven, The Netherlands (e-mail: o.raz@tue.nl). a simple passive splitting and a more advanced WDM based filtering, in this way allowing network reconfigurability while maintaining backwards compatibility with existing infrastructure. Since traditionally PON splitter boxes are located in cabinets where no electric power is present, power supply based on photo-voltaic cells was suggested.

In an attempt to implement a true packet switch rather than a network reconfigurability system, PLZT switches [6] (Lead Lanthanum Zirconate Titanate) were used. While switching speeds of 10 ns are possible, their insertion losses and power consumption are very high. Furthermore, only unicast is possible, which is a drawback when also broadcast services like television have to be provided to multiple users on the same network.

Previously [7], we have suggested to use membrane InP switches (MIPS) on the silicon-on-insulator (SOI) waveguide platform to implement a remotely controlled all-optical packet switch. In this work, a demonstration of a single MIPS showed that switching with high extinction ratio (ER) at high speed ( $<1 \mathrm{~ns}$ ) without any receiver sensitivity penalty was possible, however a full packet switch was not implemented. In this paper we present successful co-integration of multiple MIPS as well as other passive optical devices to make up the world's first bias-free and all-optically controlled $1 \times 4$ switch [8]. In addition we show how the MIPS concept is suitable for high bit-rate packetized communication as it allows for simultaneous clock distribution while preserving high extinction ratio switching of data.

The paper is organized as follows. In section II we give details on the overall $1 \times 4$ switch design and show separate results for the characteristic performance of the components included in the design. In section III we present experimental results showing the operation of the MIPS as a high ER switch supporting broadcast of clock signals [9]. In section IV the operation of the $1 \times 4$ switch is highlighted and in section $\mathrm{V}$ we conclude the work.

\section{SWITCH DESIGN AND FABRICATION}

\section{A. Switch Architecture}

To create a full $1 \mathrm{x} 4$ switch using the MIPS as individual switch elements, we propose to use a broadcast-and-select architecture, as depicted in the schematic in Fig. 1. In this approach, an out-of-band label is sent along with the data and a longer wavelength clock signal to the remote node. The label is then separated from the data and clock using a MachZehnder interferometer (MZI) and processed in an Arrayed 


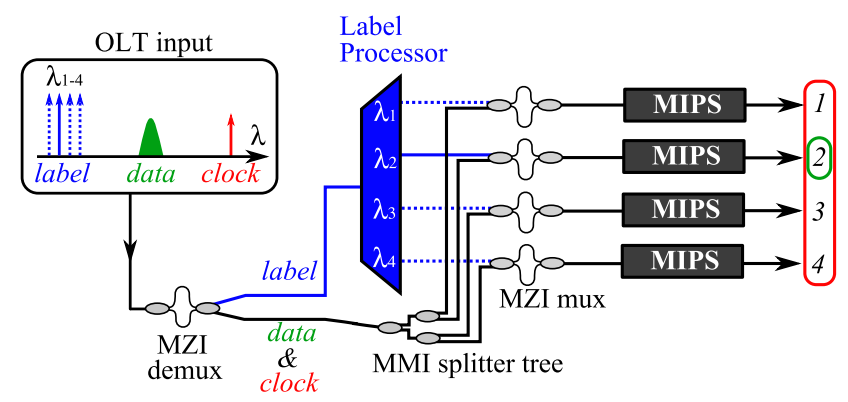

Fig. 1. Schematic of the proposed broadcast-and-select architecture. A typical input from the Optical Line Terminal (OLT) is shown as well, which results in the data appearing at port 2, while the clock is broadcasted to all ports.

Waveguide Grating (AWG), which sends the label to one of the output branches depending on the label wavelength. This is then combined again in a MZI with the broadcasted data and clock, and sent to the MIPS, which will either block or transmit the data depending on the presence of the label. The clock signal will always be transmitted however, if the wavelength is chosen longer than the device's band gap wavelength.

\section{B. Design and Characterization of the Building Blocks}

As determined in [7], the wavelengths of the labels should ideally be placed in the S-band $(\sim 1500 \mathrm{~nm})$, while the data should be put in the C-band $(\sim 1550 \mathrm{~nm})$ and the clock in the L-band (> $1600 \mathrm{~nm})$ as the device absorption drops to zero there. Therefore, the 1x2 MZI for (de)multiplexing the label and data are designed with a free spectral range (FSR) of

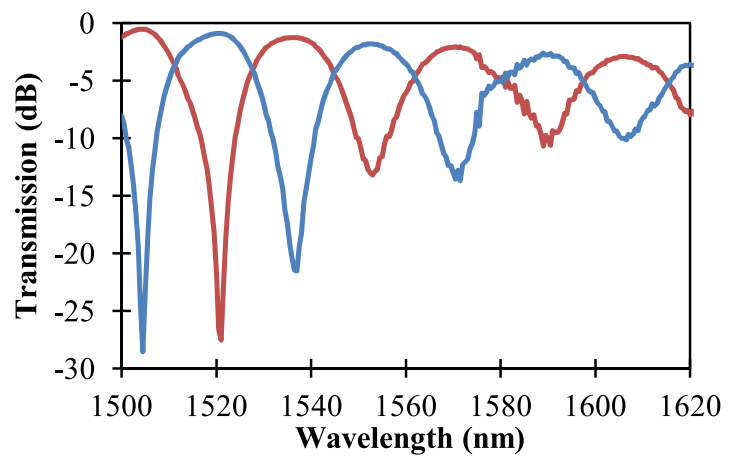

Fig. 2. Cross and through transmission of the 1x2 MZI.

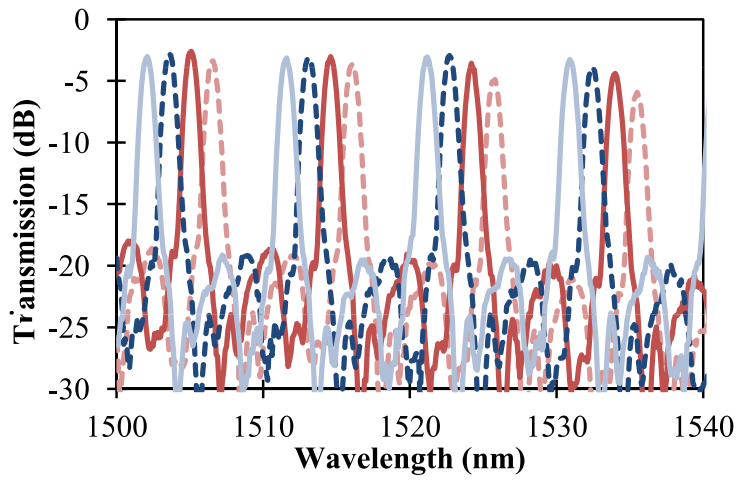

Fig. 3. Transmission spectrum of the AWG.

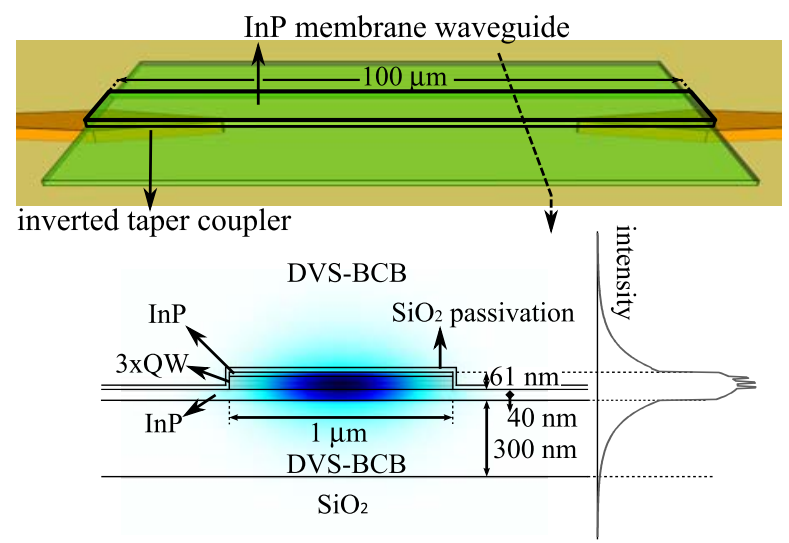

Fig. 4. MIPS layout (top) and mode profile (bottom). Due to the high index contrast, a confinement of $22 \%$ is achieved in the QWs.

$35 \mathrm{~nm}$ as this separates the data and label bands by 1.5 times the FSR, but still allows for additional freedom in case the two bands should be put closer together. Furthermore, to increase flexibility of the circuit, both arms of the MZI are equipped with a $355 \mu \mathrm{m}$ long spiral to allow a simple top heater to tune the MZI response over a full $2 \pi$ tuning range. The coupling sections of the MZI consist of a 1x2 MMI on one side and a directional coupler on the other side. The through and cross transmission response of a fabricated MZI are shown in Fig. 2. An ER of more than $10 \mathrm{~dB}$ with an insertion loss of $1-2 \mathrm{~dB}$ is obtained for wavelengths of $1500-1570 \mathrm{~nm}$. This insertion loss matches the measured insertion loss of the $1 \mathrm{x} 2 \mathrm{MMI}$.

The AWG is designed to operate at a center wavelength of $1505 \mathrm{~nm}$ with a channel spacing of $200 \mathrm{GHz}$. As the chosen AWG design does not allow for thermal tuning, the FSR is designed to be as low as $9.6 \mathrm{~nm}$ as this allows for some crude wavelength flexibility for the labels. The response of the AWG is shown in Fig. 3. The measured insertion loss is $2.5 \mathrm{~dB}$ with a worst case channel cross talk of $15 \mathrm{~dB}$, which provides sufficient channel isolation considering the strongly non-linear absorption of the MIPS.

The MIPS design is similar to the design in [7] and can be seen in Fig. 4. For the $1 \times 4$ switch experiment, the device consists of a $100 \mu \mathrm{m}$ long InP membrane ridge waveguide with a width of $1 \mu \mathrm{m}$. The active region consists of $3 \mathrm{InGaAs}$ quantum wells of $10 \mathrm{~nm}$, separated by $8 \mathrm{~nm} \mathrm{InP}$ barrier layers, and is sandwiched between two InP cladding layers. To couple the light adiabatically between the silicon waveguide circuit and the InP membrane, an $18 \mu \mathrm{m}$ long inverted taper coupler is used in which the silicon wire tapers from a width of 700 $\mathrm{nm}$ down to a tip of $100 \mathrm{~nm}$. For the clock distribution experiment a slightly different device is used: as the position of the band gap edge is critical here, an $8 \mathrm{~nm}$ InGaAs triple quantum well structure is used with a device length of $150 \mu \mathrm{m}$ and a stripe width of $2 \mu \mathrm{m}$. The spectral response of such a device is shown in Fig. 5 and shows the desired transparency in the L-band for the clock signal, while a high extinction is obtained in the C-band for the data signal for moderate onchip pumping power. The pump in this measurement was a CW beam at a wavelength of $1505 \mathrm{~nm}$, which lies in the 


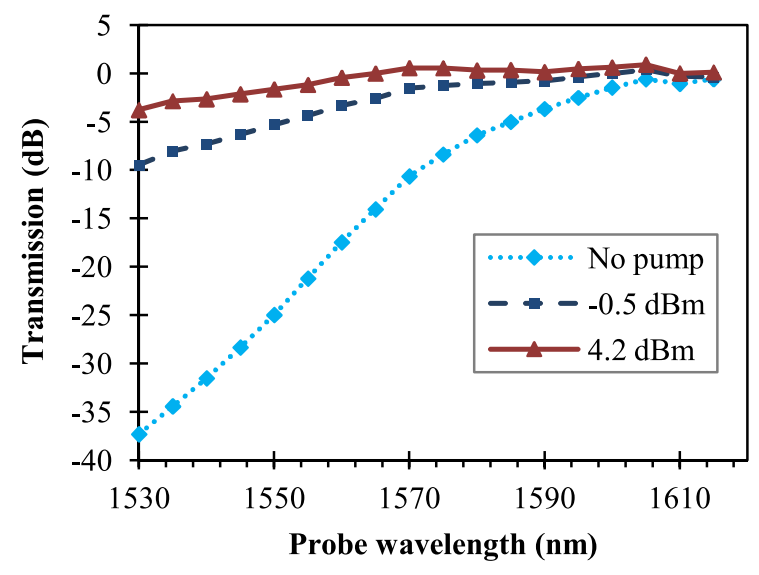

Fig. 5. Transmission spectrum of a $150 \mu \mathrm{m}$ long MIPS with $8 \mathrm{~nm}$ InGaAs quantum wells. The given pump power is the on-chip power calculated from transmission data through a reference waveguide.

wavelength range which is most suitable for the labels as determined in [7]. The reason why this quantum well stack is not used for the full $1 \mathrm{x} 4$ switch experiment as well is the poor epitaxial wafer quality of this particular wafer, which forced us to use a quantum well stack optimized for another application [10].

As a broad range of wavelengths (labels, data, and clock) should be coupled into the switch with preferably low insertion loss, the bandwidth of the grating couplers used in previous work [7] is not sufficient anymore. Therefore, a horizontal coupling scheme which uses $3 \mu \mathrm{m}$ x $3 \mu \mathrm{m}$ cleaved SU-8/BCB waveguides is used, which are coupled to the silicon waveguide circuit using $350 \mu \mathrm{m}$ long inverted taper couplers which taper from $100 \mathrm{~nm}$ tips to a $450 \mathrm{~nm}$ broad silicon wire. The coupling loss from fiber to chip was determined to be 3.5-4.0 dB over a wavelength range of 1500 $1620 \mathrm{~nm}$.

\section{Fabrication details}

The $220 \mathrm{~nm}$ thick silicon waveguide circuit is fabricated using a $193 \mathrm{~nm}$ deep UV lithography stepper and dry etching on a $200 \mathrm{~mm}$ wafer in a CMOS pilot-line [11]. After dicing of the SOI wafer, the separate dies are cleaned in a standard clean-1 solution for 15 minutes. At the same time, the III-V provided by a commercial manufacturer is cleaved, and separate dies are prepared for bonding by wet chemical etching of an InP/InGaAs sacrificial layer pair. To improve

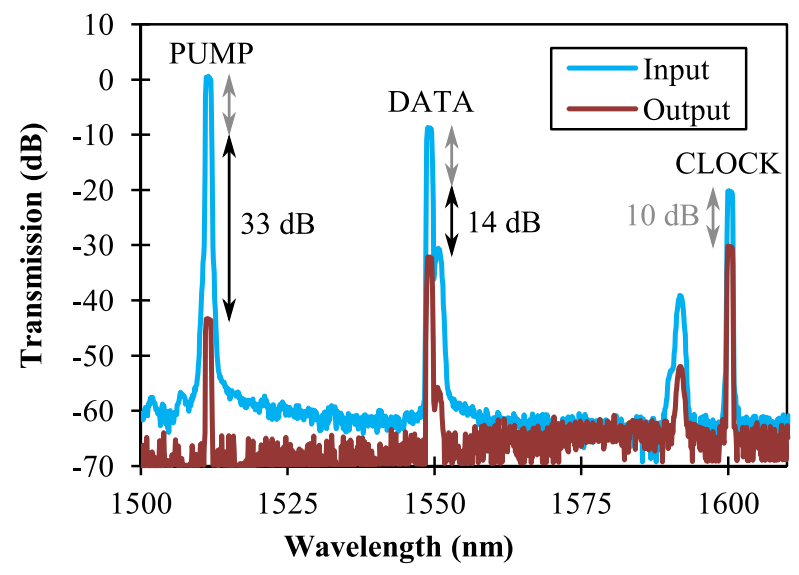

Fig. 7. Spectrum at the input and output of the MIPS. The loss due to fiber-to-chip coupling is marked with a grey arrow, while the loss due to MIPS absorption is marked with a black arrow.

adhesion to the DVS-BCB adhesive layer, a thin layer of 15 $\mathrm{nm}$ of $\mathrm{SiO}_{2}$ is deposited on the III-V dies. After this, a DVSBCB solution diluted with mesitylene (1BCB:1Mes) is spincoated on the SOI dies. After evaporation of the mesitylene, the dies are brought into close contact in a controlled environment using a bonding pressure of 1.25MPa, and cured for one hour at $240^{\circ} \mathrm{C}$ [12]. After the bonding of the dies, the InP substrate is removed using a combination of mechanical grinding and wet chemical etching using $\mathrm{HCl}$, until the InGaAs etch stop layer is reached. Subsequently the etch stop layer is selectively removed using a $1 \mathrm{H}_{2} \mathrm{SO}_{4}: 1 \mathrm{H}_{2} \mathrm{O}_{2}: 18 \mathrm{H}_{2} \mathrm{O}$ solution and a resist mask is spincoated and patterned using contact lithography to create the core of the waveguide. By subsequent $\mathrm{HCl}$ and $1 \mathrm{H}_{2} \mathrm{SO}_{4}: 1 \mathrm{H}_{2} \mathrm{O}_{2}: 18 \mathrm{H}_{2} \mathrm{O}$ wet etching of the quantum wells and barrier layers, the waveguide core is defined. Using a second, broader resist mask and subsequent $\mathrm{HCl}$ wet etching also the bottom InP layer can be etched. After this, the MIPS is passivated by depositing a $15 \mathrm{~nm} \mathrm{SiO}$ layer and spinning and curing a 1.2 $\mu \mathrm{m}$ thick BCB cladding layer on top of the sample. Using two subsequent metallization steps, both the heater $(100 \mathrm{~nm} \mathrm{Ti})$ and conducting lines and pads ( $300 \mathrm{~nm} \mathrm{Au}$ ) can be defined. To couple the circuit to the outside world, the BCB cladding is first etched back $600 \mathrm{~nm}$ near the edges of the device, where the silicon inverted tapers for horizontal coupling are defined. Subsequently $2.4 \mu \mathrm{m}$ thick SU-8 waveguides are defined on top of these inverted tapers, leading to a combined BCB and

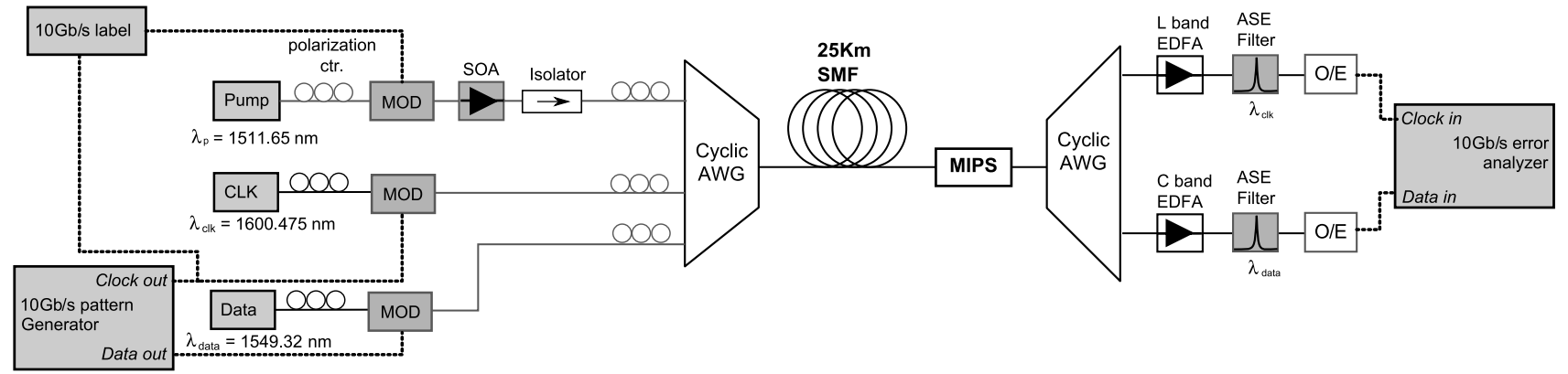

Fig. 6. Setup for the proof-of-principle clock distribution experiment. 


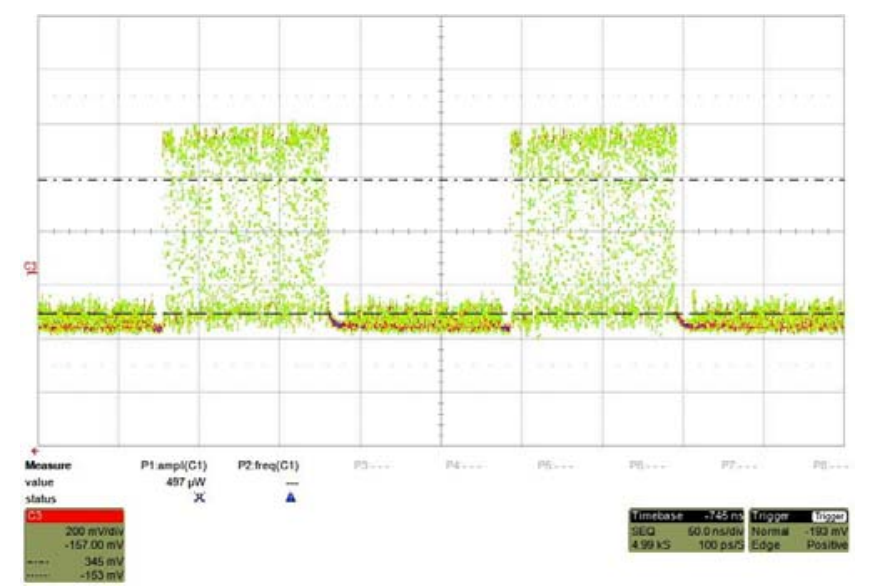

Fig. 8. Detected data signal at the AC receiver. One out of two packets is being blocked by the MIPS.

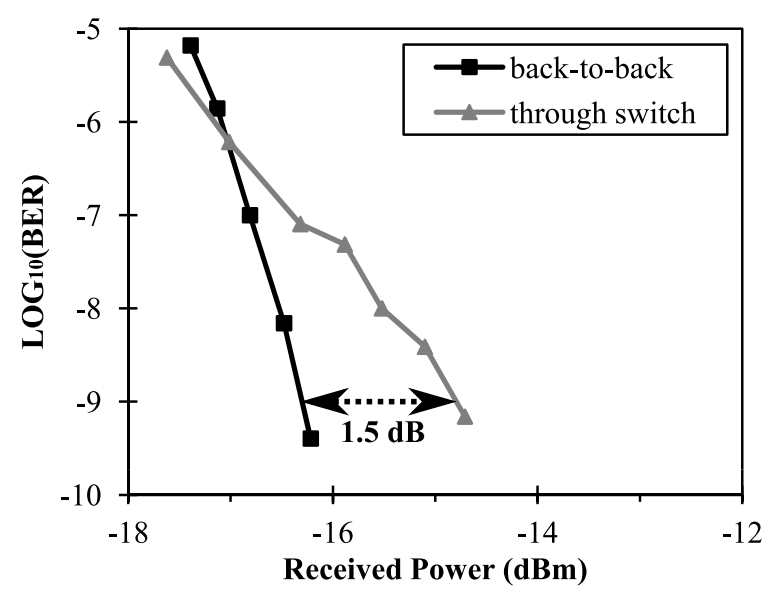

Fig. 9. BER as a function of received power.

SU-8 thickness of $3 \mu \mathrm{m}$, which matches the mode size of the lensed fibers used in the measurement setup. Finally, after thinning down the silicon substrate, the dies are cleaved.

\section{Clock Distribution EXPERIMENT}

\section{A. Setup}

In a first experiment, a single MIPS is placed in a labversion of a typical fiber-to-the-home network, as a proof-ofconcept experiment for clock distribution using the MIPS as a building block for an all-optical packet switch (Fig. 6). At the transmission side, the central office combines a data, clock and pump signal using a cyclic AWG. The data signal consists of packets of $2^{10}-1$ pseudorandom bit sequences (PRBS) with a guard time in between packets of 50 bits ( $5 \mathrm{~ns})$. The generated packets are modulated at a bit rate of $10 \mathrm{~Gb} / \mathrm{s}$ on a laser with a wavelength of $1549.32 \mathrm{~nm}$ using non-return-tozero (NRZ) on-off-keying (OOK). The clock signal is modulated using the clock output of the data pattern generator on a laser with a wavelength of $1600.475 \mathrm{~nm}$. The pump signal which is at a wavelength of $1511.65 \mathrm{~nm}$ is modulated in such a way that it is only on during one out of two packets. This is done using a second pattern generator that uses the same clock signal to operate in sync. These signals are then sent through a spool of $25 \mathrm{~km}$ of fiber. At the input of the MIPS, the pump, data and clock signals have a power of $0 \mathrm{dBm},-9 \mathrm{dBm}$ and $-20 \mathrm{dBm}$ respectively. In Fig. 7, the input and output spectrum is shown. Due to a relatively high fiberto-chip coupling loss of $5 \mathrm{~dB}$ per facet of the SU-8 couplers on this particular sample (see section II.B), the on-chip pump power was insufficient to completely bleach the absorption for the data signal. Note that the secondary peaks in the spectrum are made up of filtered ASE of the SOA for the pump beam by the cyclic AWG. At the receiver side, the transmitted signals are being split again using a cyclic AWG, so the presence of these peaks has no effect on the detection of data and clock signals. Due to a relatively high loss of the device, the output signal levels for data and clock both had to be amplified using two EDFA pre-amplifiers, which deteriorated the opticalsignal-to-noise-ratio (OSNR) of these signals. After filtering of the ASE, the signals are both sent to an AC coupled detector. The detected clock signal is additionally filtered using a high-Q OC-192 RF filter and injected together with the detected data signal in the error analyzer to analyze the performance of the optical link. The use of AC coupled detectors is the reason why the data packet length was restricted to about $100 \mathrm{~ns}$, in order to avoid AC coupled eye closure during a switched-off packet in the error analyzer.

\section{B. Results}

In Fig. 8 the data signal as detected by the AC coupled detector is shown after transmission through the MIPS. Due to the fact that the pump signal is only on during one out of two packets, the MIPS is only transmitting one out of two data packets. However, the achieved ER is much lower than what is potentially possible (as can be seen in Fig. 5). This is due to a combination of the lack of power to bleach the device completely and the limited ER in the modulation of the pump signal. In a real application, seeing the very slow modulation speed needed (5-10 MHz), direct current modulation can be used to completely turn off the pump signal thereby improving the undesirable cross talk during the off state.

In Fig. 9 the bit error rate (BER) performance of the link is shown. The measured performance takes into consideration the extra $3 \mathrm{~dB}$ peak-to-average power ratio of the partially transmitted data stream. At a BER of $10^{-9}$ there is a power penalty of $1.5 \mathrm{~dB}$. This penalty is caused by the poor ER of the switch in this experiment as explained above, in combination with a reduced OSNR due to the required amplification by an EDFA after the switch. The fact that the slope of the BER curves differs, is related to the reduced OSNR as well. This can be understood from equation (1), which is reproduced from [13].

$$
\delta_{I}=-10 \log _{10}\left(1-r_{I}^{2} Q^{2}\right)
$$

This equation relates the power penalty due to intensity noise $\delta_{I}$ to the $Q$ parameter and the intensity noise parameter $r_{I}$ and shows that the slope $d \delta_{I} / d Q$ increases with increasing intensity noise $r_{I}$. An uncommon feature in the BER curves is however that the back-to-back curve crosses the curve for 


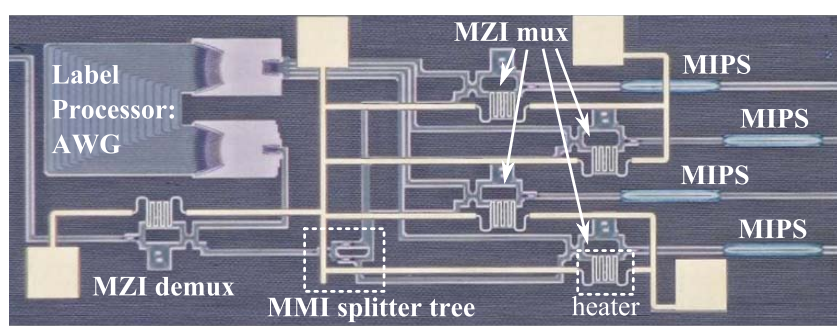

Fig. 10. Optical microscopy image of a fabricated $1 \times 4$ switch.
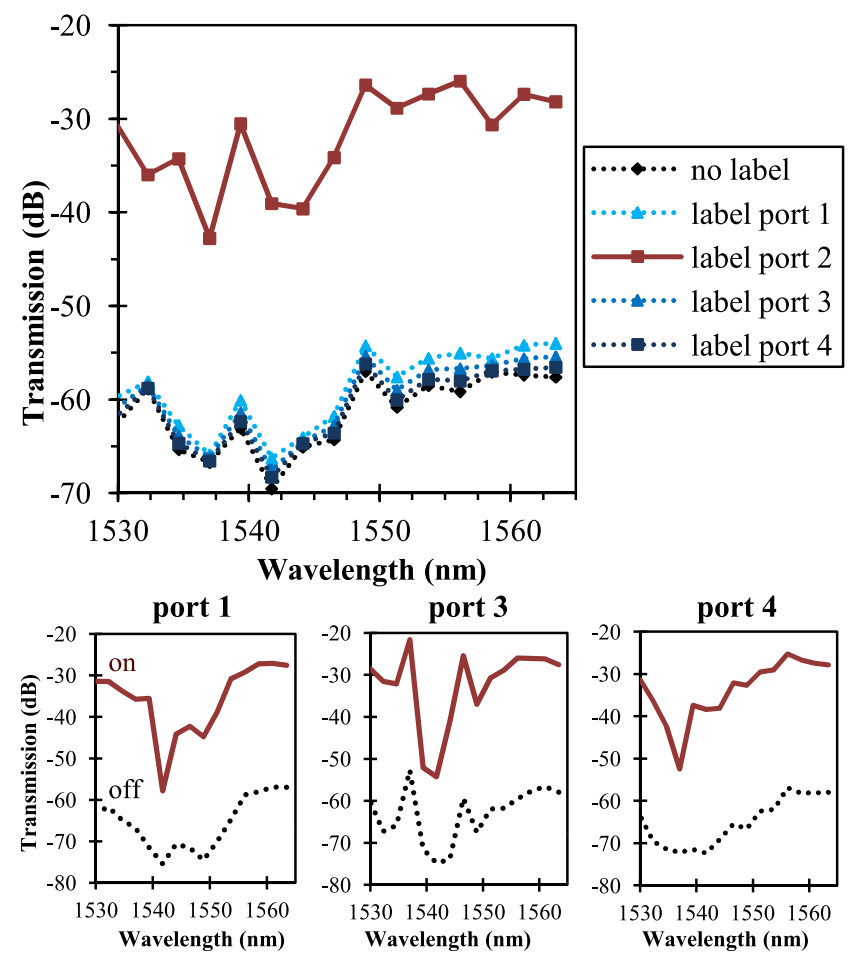

Fig. 11. Static transmission of the switch at output port 2 with label corresponding to the different output ports. From the comparison with the case where the label is off, we can conclude that the switch handles the cross talk due to the MZIs and AWG very well. At the bottom, also the transmission for the other output ports are shown.

transmission through the MIPS at high bit error rates. This seems to be related to the fact that the device is not completely bleached in its 'on' state. Therefore, the device transmission for the data signal will be non-linear, causing the device to operate as a signal regenerator [10]. As a consequence, the ER of the data signal will be slightly improved and the corresponding power penalty at the receiver reduced, which leads to the crossing of the BER curves. It is important to mention that the MIPS does not inflict any pattern dependence as we have previously reported [7].

Although the device in this experiment suffered from a too high insertion loss in both setup and device causing the power penalty in the BER measurement, the usefulness of a transparent wavelength band for clock distribution could be shown, as clock recovery at the receiver could in this way be avoided.

\section{SWITCHING EXPERIMENT}

\section{A. Static performance}

Next to a single MIPS, also a full $1 \mathrm{x} 4$ switch was fabricated. An optical microscopy image can be seen in Fig. 10. In a first experiment the static performance of the switch was characterized by injecting both a CW label corresponding to one of the output channels and a CW data signal for which the wavelength was swept over the C-band. In this way, the intrinsic ER of the switch can be obtained as well as the effect of the cross talk in the AWG and MZIs. The label power was 10.6 $\mathrm{dBm}$, and the data signal power was $-0.6 \mathrm{dBm}$ before coupling into the chip. In Fig. 11 we show the behavior at a single output port of the switch when alternating the label wavelength between the different output port labels. The used label wavelengths were at $1510.77 \mathrm{~nm}, 1509.22 \mathrm{~nm}, 1507.80$ $\mathrm{nm}$ and $1506.35 \mathrm{~nm}$ for ports 1-4 respectively. In the inset, also transmission at the other output ports is shown. A high port isolation of more than $25 \mathrm{~dB}$ could be achieved in all the output ports across the C-band, up to more than $30 \mathrm{~dB}$ for 1550-1560 nm, the intended wavelength range for the data signal. Furthermore, tuning the label wavelength to another output port nearly has the same effect on the transmission of the data signal as turning the label power off. Although the cross talk levels in the passive components (AWG and MZIs) are much worse than $30 \mathrm{~dB}$, the non-linear absorption in the MIPS reduces its effect on the actual device cross talk.

The achieved insertion loss is of the order of $26 \mathrm{~dB}$. There are several causes for this loss. A first cause is the fiber-tochip coupling loss which was determined to be $3.5 \mathrm{~dB}$ per transition. Furthermore the passive components used in the switch architecture were not ideal, and excess losses due to MMIs and MZIs for the data signal accumulated to $4 \mathrm{~dB}$. Obviously also the broadcasting of the data signal yields a loss of $6 \mathrm{~dB}$. The remainder of the loss $(9 \mathrm{~dB})$ can be attributed to scattering losses and insufficient pumping of the MIPS devices.

\section{B. Dynamic performance}

To demonstrate the operation of the switch, also a BER experiment was performed. The setup that was used is depicted in Fig. 12. To achieve a better ER in the label than in the clock distribution experiment, the label is modulated by directly modulating a C-band SOA, as the label can be completely turned off in this way. To better exploit the gain of the C-band SOA that was used to modulate the label, a different set of label wavelengths was chosen in comparison with the static measurement. The labels were put at 1535, 1536.6, 1538.2 and $1539.8 \mathrm{~nm}$. The data signal was created by modulating a laser at $1559.38 \mathrm{~nm}$ using with a $10 \mathrm{~Gb} / \mathrm{s}$ pattern generator running a $2^{31}-1$ PRBS using a NRZ OOK modulation scheme. Due to the fact that this initial design still suffers from too high insertion losses, both the data signal and label had to be amplified using an EDFA to boost the input signal power to 4.5 and $13 \mathrm{dBm}$ respectively. After combining data and label in an AWG, the signals are sent through the $1 \times 4$ switch. In the switch, the heaters were tuned to align the MZI 


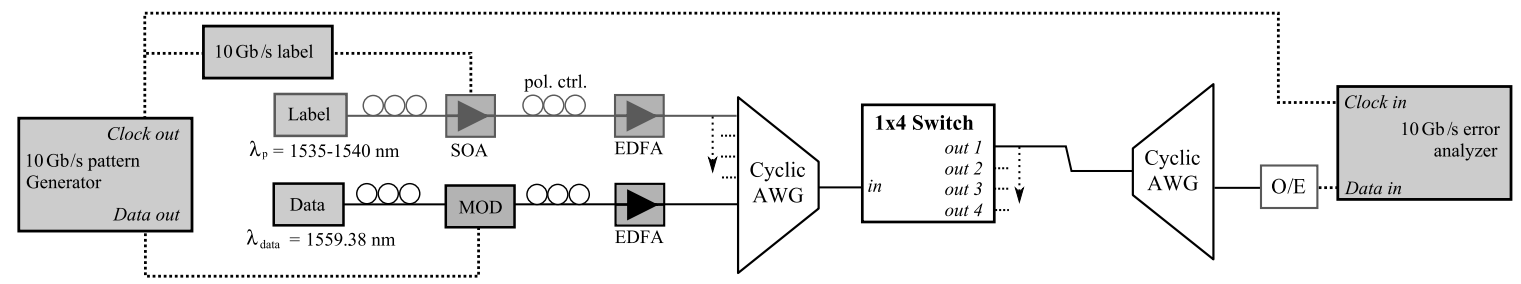

Fig. 12. Schematic of the test setup for the $1 \mathrm{x} 4$ switch. As marked by the dotted arrows, the output fiber has to be moved to measure the different output ports, and the input fiber with the label has to be switched to the input corresponding to the right wavelength of the input cyclic AWG.

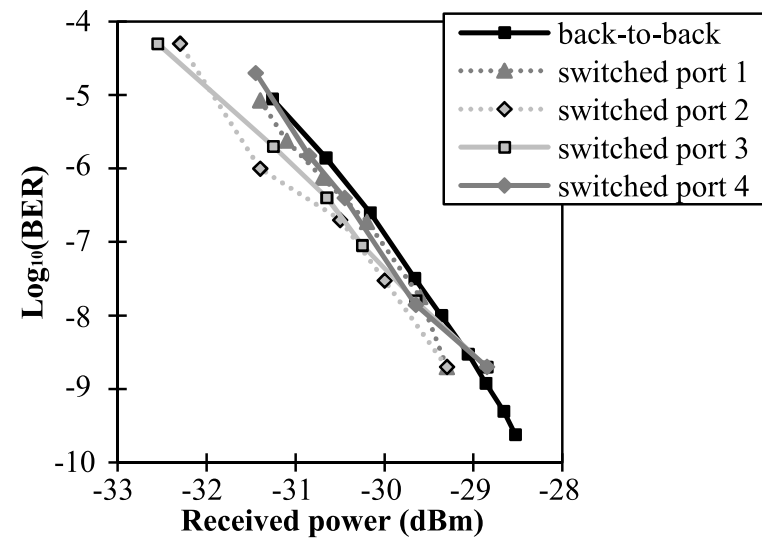

Fig. 13. BER for a PRBS transmitted through the different ports of the switch. There is no measurable power penalty.

filter curves to the label and data bands. At the output, the light is split again using an AWG, and the data signal is detected by a detector and sent to the BER analyzer. The receiver used for these measurements was an AC coupled avalanche photodiode housed inside an XFP module with a typical receiver sensitivity of $-28 \mathrm{dBm}$.

In Fig. 13 the measured BER is shown for a PRBS routed through each of the output ports, while keeping the label operating in CW. There is no measurable power penalty related to the transmission through the switch. Since the detector used in this experiment was AC coupled, it could not be used to measure the BER for packetized data. Therefore the AC coupled detector was replaced by a DC coupled Agilent 8192A $10 \mathrm{~Gb} / \mathrm{s}$ receiver. Also in this case, error free operation could be achieved, with a penalty of $0.8 \mathrm{~dB}$. In addition we show a time trace together with the eye diagrams of the packetized data for the back-to-back case and at the output of port 1 in Fig. 14. The label is modulated in such a way that only one out of four packets is being transmitted. The resulting time trace clearly shows the high ER that can be obtained with the switch. The eye diagrams showing the packetized data were captured with the DC coupled Agilent receiver. From these, we can see that except for some additional noise both eyes are identical and open, confirming the result obtained with the BER measurement. For the eye after transmission through the switch, the optical input power at the receiver was much lower $(-18.5 \mathrm{dBm}$ compared to $-10.2 \mathrm{dBm}$ in the backto-back case), which explains the increased electrical noise in the detected signal.

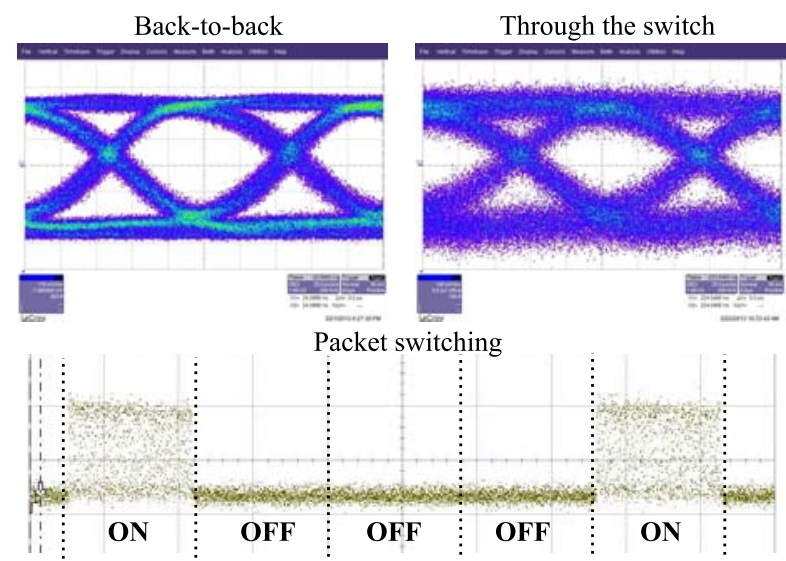

Fig 14. (Top) Eyes for the data signal in the back-to-back measurement and switched to port 1 for the packetized data as detected by the Agilent DC coupled receiver. (Bottom) Time trace displaying the operation of the switch, where only 1 in 4 packets is being transmitted.

\section{Future improvements}

Although successful switching was demonstrated, the fabricated device suffered from considerable insertion losses, which currently limits the applicability of the device. It is expected that optimization of the different components can eliminate most of the excess loss however.

Most of the loss in the passive components can be avoided if the current state of the art passives are used. MMIs [14] have reached excess losses of only $0.06 \mathrm{~dB}$ at $1550 \mathrm{~nm}$ and remain limited to less than $0.2 \mathrm{~dB}$ in the band of $1500 \mathrm{~nm}$ to $1600 \mathrm{~nm}$. AWGs [15] have been demonstrated with an insertion loss of less than $1 \mathrm{~dB}$. Inverted taper couplers using a cleaved SU-8 waveguide for fiber-to-chip coupling have reached a coupling loss of only $0.66 \mathrm{~dB}$ [16]. These improvements reduce both insertion loss for data and clock signal, and the required label power to drive the MIPS.

Next to improving the passive components, also the fabrication of the MIPS can be optimized. By further reducing the width of the devices from $1 \mu \mathrm{m}$ to $700 \mathrm{~nm}$, the amount of power required to pump the device can be reduced by $30 \%$, while the confinement of light in the active region and therefore device operation remains nearly the same. High CW gain in devices with in-well optical pumping [17] suggests furthermore that improved epitaxy and processing of the MIPS should allow us to generate gain in the devices. To that end, we are currently exploring sulfur passivation techniques [18] to increase the carrier lifetime of the devices, which would reduce both power consumption and heating, the probable cause for the lack of gain [19]. 


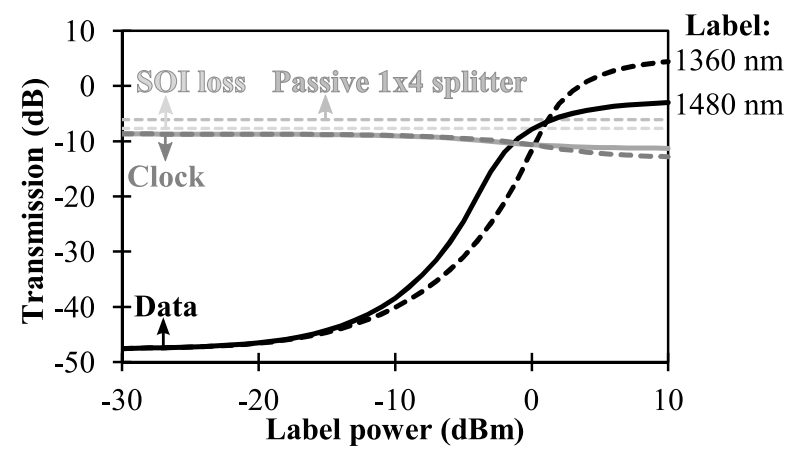

Fig. 15. Simulated transmission of a data $(1550 \mathrm{~nm}$, black $)$ and clock (1610 nm, grey) signal through an optimized design with a label at 1480 $\mathrm{nm}$ (solid lines) and $1360 \mathrm{~nm}$ (dashed lines). As a reference, also the accumulated loss of the passives at $1550 \mathrm{~nm}$ is shown, and the loss at 1550 $\mathrm{nm}$ in the case a simple passive splitter tree would be used.

To quantify the possible performance of an improved device, a simulation was performed. For the passive components the characterization results from [14-16] were used. For the MIPS, the device transmission was simulated using a previously developed simulation engine [10]. To account for the improved passivation a carrier lifetime of 1.5 ns was assumed [20]. In [10] the measured absorption was fitted to extract the quantum well response as a function of carrier density. However, because of an anticipated reduced heating of the device, this data is not valid anymore, and is therefore replaced by a numerically simulated quantum well response at $300 \mathrm{~K}$, using the method as described in [21].

In Fig. 15 the simulated switch response of a $1 \times 4$ switch with $150 \mu \mathrm{m}$ long MIPS devices can be seen under two pumping conditions. From this it can be seen that device performance is much better: the MIPS is already bleached at a off-chip label power of $0 \mathrm{dBm}$, while for a higher power the additional gain provided by the MIPS compensates for insertion and splitter losses. The gain that can be achieved is strongly dependent on the label wavelength however, as the highest free carrier density that can be generated for a particular label wavelength is the transparency carrier density, which is higher for shorter wavelengths.

\section{CONCLUSION}

We have demonstrated an all-optical $1 \times 4$ reconfigurable remote node switch, based on the integration of the MIPS in a broadcast-and-select architecture implemented in a SOI circuit. It was shown that an ER of more than $25 \mathrm{~dB}$ could be achieved and that furthermore for CW labels data could be sent without power penalty at the receiver. Furthermore, we have shown that the inherent transparency for longer wavelengths of the MIPS can be useful to allow broadcasting of signals along the network, without the need for optical control. This was demonstrated by sending a clock signal through a network link, allowing for data detection without needing clock recovery. Such a broadcasting possibility could also be useful to provide backwards compatibility with OLTs and ONUs configured for PON operation.

The fabricated chips suffered from considerable insertion losses, which complicated the experiments presented in this paper. However, we have shown that optimization of passives and MIPS fabrication would lead to much better device characteristics.

\section{REFERENCES}

[1] (2013). Cisco Visual Networking Index: Forecast and Methodology, 2012-2017 [Online]. Cisco Systems, USA. Available: http://www.cisco.com/en/US/solutions/collateral/ns341/ns525/ns537/ns7 05/ns827/white paper c11-481360.pdf

[2] E. Wong, "Next-Generation Broadband Access Networks and Technologies." J. Lightwave Technol., vol. 30, nr. 4, pp. 597-608, 2012.

[3] J. Theodoras, and K. Grobe (2010). 100GbE - the Future of Ethernet. ADVA Optical Networking, Germany [Online]. Available: http://walkerfirst.com/uploads/files/literature/ADVA\%20100GbE.pdf

[4] J.H. Lee, K.-M. Choi, and C.-H. Lee, "A remotely reconfigurable remote node for next-generation access networks," IEEE Phot. Tech. Lett., vol. 20, nr. 11, pp. 915-917, 2008.

[5] H. Ramanitra, P. Chanclou, Z. Belfqih, M. Moignard, H. Le Bras, and D. Schumacher, "Scalable and multi-service passive optical access infrastructure using variable optical splitters," in Proc. OFCNFOEC, 2006, pp. 2391-2393.

[6] Y. Hara, T. Sato, Y. Shimada, K. Ashizawa, K. Tokuhashi, D. Ishii, S. Okamoto, and N. Yamanaka, "A study of next generation metro-access hybrid scalable network by using PLZT ultra high speed optical wavelength selective switch," in Proc. ISAS, 2011, pp. 1-6.

[7] M. Tassaert, G. Roelkens, H. Dorren, D. Van Thourhout, and O. Raz, "Bias-free, low power and optically driven membrane InP switch on SOI for remotely configurable photonic packet switches,” Opt. Express, vol. 19, pp. B819-B824, 2011.

[8] O. Raz, M. Tassaert, G. Roelkens, and H.J.S. Dorren, "Optically reconfigurable $1 \times 4$ silicon-on-insulator remote node switch for access networks," in Proc. OFCNFOEC, 2013, pp. PDP5C.2.

[9] O. Raz, M. Tassaert, G. Roelkens, and H.J.S. Dorren, "All-optical membrane InP switch on silicon for access applications", in Proc. ACP, 2012, pp. AS1B5.

[10] M. Tassaert, H.J.S. Dorren, G. Roelkens, and O. Raz, "Broadband passive InP membrane regenerator for silicon-based optical interconnects applications," in Proc. OFCNFOEC, 2013, pp. OW3D.4.

[11] Epixfab. Available: http://www.epixfab.eu/

[12] G. Roelkens, J. Brouckaert, D. Van Thourhout, R. Baets, R. Notzel, and M. Smit, "Adhesive bonding of InP/InGaAsP dies to processed siliconon-insulator wafers using dvs-bis-benzocyclobutene," J. Electrochem. Soc., Vol. 153, pp. G1015-G1019, 2006.

[13] G.P. Agrawal, "Optical Receivers," in Fiber-optical communication systems, 3th ed. New York: John Willey \& Sons, 2002, pp. 169-170.

[14] Z. Sheng, Z. Wang, C. Qiu, L. Li, A. Pang, A. Wu, X. Wang, S. Zou, and F. Gan"A compact and low-loss MMI coupler fabricated with CMOS technology." IEEE Phot. J., Vol. 4, nr. 6, pp.2272-2277, 2012.

[15] S. Pathak, D. Van Thourhout, and W. Bogaerts, "An optimized design of SOI-based AWGs for (de)multiplexer applications," submitted for publication in Opt. Letters.

[16] M. Pu, L. Liu, H.Y. Ou, K. Yvind, and J.M. Hvam, "Ultra-low-loss inverted taper coupler for silicon-on-insulator ridge waveguide," Optics Comm., vol. 283, pp.3678-3682, 2010.

[17] S.S. Beyertt, M. Zom, T. Kubler, H. Wenzel, M. Weyers, A. Giesen, G. Trankle, and U. Brauch, "Optical in-well pumping of a semiconductor disk laser with high optical efficiency," IEEE J. Quant. Electron., vol. 41, no. 12, pp.1439-1449, 2005.

[18] D. Sheela, and N. DasGupta, "Optimization of surface passivation for InGaAs/InP pin photodetectors using ammonium sulfide," Semicond. Sci. Technol., vol. 23, no. 3, pp. 035018, 2008.

[19] M. Tassaert, S. Keyvaninia, D. Van Thourhout, W.M.J. Green, Y. Vlasov, and G. Roelkens, "An optically pumped nanophotonic InP/InGaAlAs optical amplifier integrated on a SOI waveguide circuit," Opt. and Quantum Electron., vol. 44, no. 12, pp.513-519, 2012.

[20] J.E. Ehrlich, D.T. Neilson, A.C. Walker, G.T. Kennedy, RS. Grant, W. Sibbett, and M. Hopkinson. "Carrier lifetimes in MBE and MOCVD InGaAs quantum wells." Semicond. Sci. Technol., Vol. 8, nr. 2, pp. 307309, 1993.

[21] M. Tassaert, D. Van Thourhout, and G. Roelkens, "Strategies to increase the modal gain in heterogeneously integrated III-V amplifiers on Silicon-On-Insulator," Opt. and Quantum Electron., Vol. 44, nr. 14, p.683-689 (2011) 